\title{
Biomechanical analysis of smart walking shoe sending movement information to display device by radio communication
}

\author{
Seung-Bum Park, Kyung-Deuk Lee, Dae-Woong Kim, Jung-Hyeon Yoo, Kyung-Hun Kim \\ From 4th Congress of the International Foot and Ankle Biomechanics (i-FAB) Community \\ Busan, Korea. 8-11 April 2014
}

The purpose of this study was to find the difference in foot pressure patterns when wearing smart walking shoes. Foot pressure measurement is an established tool for the evaluation of foot function [1]. These measurements assess the effect of structural changes, which may occur as a complication of pathologies such as diabetes, and therefore have been suggested as one of the key tools in ulcer risk estimation [2].

The subjects who took part in the test consist of 5 elderly people and 5 young people. The physical features of the elderly people that were recruited for the study are shown below: 5 healthy male subjects (elderly people) with an average age of $62.0 \mathrm{yrs}$ (S.D $1.0 \mathrm{yrs)}$ ), weight of $69.4 \mathrm{~kg}$ (S.D $10.0 \mathrm{~kg}$ ), height of $168.8 \mathrm{~cm}($ S.D $5.3 \mathrm{~cm})$ and a foot size of $270.0 \mathrm{~mm}$ (S.D $0.0 \mathrm{~mm}$ ). 5 healthy male subjects (young people) with an average age of 27.2 yrs (S.D 4.1 yrs), weight of $75.2 \mathrm{~kg}$ (S.D $4.6 \mathrm{~kg}$ ), height of $175.4 \mathrm{~cm}$ (S.D $4.0 \mathrm{~cm}$ ) and a foot size of $270.0 \mathrm{~mm}($ S.D $0.0 \mathrm{~mm}$ ). Ten males ( 5 elderly people, 5 young people) walked on a treadmill wearing three different shoes. Foot pressure data (Contact areas, Maximum forece, Peak pressure, Maximum mean pressure) was collected using a Pedar-X mobile system (Novel Gmbh., Germany) operating at the $1,000 \mathrm{~Hz}$.

The results are as follows:

1. Young people

In comparison with the Type B (control shoes):

1) Type A (development shoes)

a) The contact area of foot (Total) by increased $8.36 \%$, forefoot (M1) by increased $8.95 \%$, midfoot (M2) by increased $12.18 \%$ and rearfoot (M3) by increased $4.48 \%$. b) The maximum force of foot (Total) by decreased $4.02 \%$,

\footnotetext{
* Correspondence: sbpark@shoenet.org

Footwear Biomechanics Team, Footwear Industrial Promotion Center, Busan,
} Korea

rearfoot (M3) by decreased $6.39 \%$, while the maximum force of forefoot (M1) by increased $2.48 \%$ and midfoot (M2) by increased $17.52 \%$. c) The peak pressure of foot (Total) by increased $2.28 \%$, forefoot (M1) by increased $6.19 \%$, while the peak pressure of midfoot (M2) by decreased $2.91 \%$ and rearfoot (M3) by decreased $13.69 \%$. d)The maximum mean pressure of foot (Total) by decreased $12.74 \%$, forefoot (M1) by decreased $6.90 \%$, midfoot (M2) by decreased $2.79 \%$ and rearfoot (M3) by decreased $11.18 \%$.

2) Type $C$ (smart walking shoes)

a) The contact area of foot (Total) by increased $7.96 \%$, forefoot (M1) by increased $8.90 \%$, midfoot (M2) by increased $11.81 \%$ and rearfoot (M3) by increased $3.50 \%$. b) The maximum force of foot (Total) by decreased $5.27 \%$, forefoot (M1) by decreased $0.67 \%$ and rearfoot (M3) by decreased $5.67 \%$, while the maximum force of midfoot (M2) by increased $23.55 \%$. c) The peak pressure of foot (Total) by decreased 6.70\%, forefoot (M1) by decreased $3.35 \%$ and rearfoot (M3) by decreased $10.54 \%$, while the peak pressure of midfoot (M2) by increased $2.19 \%$. d) The maximum mean pressure of foot (Total) y decreased $10.97 \%$, forefoot (M1) by decreased $7.62 \%$, midfoot (M2) by decreased $1.15 \%$ and rearfoot (M3) by decreased $8.02 \%$.

2. Elderly people

In comparison with the Type B (control shoes):

1) Type A (development shoes)

a) The contact area of foot (Total) by increased $8.09 \%$, forefoot (M1) by increased 5.47\%, midfoot (M2) by increased $22.66 \%$ and rearfoot (M3) by increased $3.21 \%$. b) The maximum force of foot (Total) by decreased $2.13 \%$, forefoot (M1) by decreased $3.53 \%$ and rearfoot (M3) by decreased $9.85 \%$, while the maximum force of midfoot (M2) by increased $41.32 \%$. c) The peak pressure 


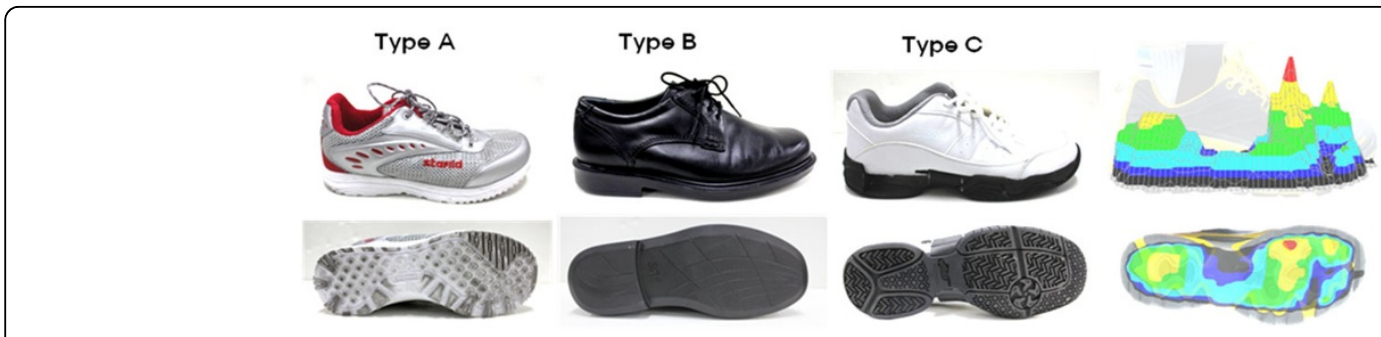

Figure 1 Type A: development shoes, Type B: control shoes, Type C: smart walking shoes

Table 1 Result of Foot Pressure

\begin{tabular}{cccccccc}
\hline Subjects & Mask & \multicolumn{3}{c}{ Contact area $\left(\mathbf{c m}^{\mathbf{2}}\right)$} & \multicolumn{3}{c}{ Maximum force (N) } \\
\cline { 3 - 7 } & & $\mathbf{A}$ & $\mathbf{B}$ & $\mathbf{C}$ & $\mathbf{A}$ & $\mathbf{B}$ & $\mathbf{C}$ \\
\hline Young & Total & $142.877 \pm 6.584$ & $131.852 \pm 10.934$ & $142.342 \pm 5.754$ & $711.105 \pm 59.923$ & $740.921 \pm 95.996$ & $701.841 \pm 60.198$ \\
& M1 & $68.663 \pm 1.716$ & $63.023 \pm 5.373$ & $68.629 \pm 0.584$ & $621.023 \pm 89.605$ & $606.018 \pm 168.64$ & $601.982 \pm 86.053$ \\
& M2 & $33.443 \pm 5.540$ & $29.811 \pm 4.185$ & $33.331 \pm 5.175$ & $133.911 \pm 8.162$ & $113.943 \pm 21.044$ & $140.778 \pm 14.482$ \\
& M3 & $40.770 \pm 0.000$ & $39.019 \pm 2.200$ & $40.383 \pm 0.753$ & $468.385 \pm 42.442$ & $500.382 \pm 46.850$ & $471.992 \pm 27.290$ \\
\hline Elderly & Total & $139.403 \pm 2.996$ & $128.966 \pm 5.757$ & $138.099 \pm 4.256$ & $592.178 \pm 95.362$ & $605.047 \pm 81.495$ & $596.161 \pm 100.23$ \\
& M1 & $68.119 \pm 3.213$ & $64.589 \pm 5.796$ & $68.221 \pm 3.705$ & $526.524 \pm 75.498$ & $545.776 \pm 74.082$ & $546.801 \pm 90.669$ \\
& M2 & $30.514 \pm 2.751$ & $24.877 \pm 5.708$ & $29.140 \pm 4.599$ & $110.238 \pm 25.983$ & $78.007 \pm 31.900$ & $96.843 \pm 29.870$ \\
& M3 & $40.770 \pm 0.000$ & $39.503 \pm 1.290$ & $40.736 \pm 0.060$ & $386.392 \pm 94.017$ & $428.618 \pm 84.020$ & $397.017 \pm 94.609$ \\
\hline Subjects & Mask & & Peak pressure (kPa) & & Maximum mean pressure (kPa) \\
& & & $\mathbf{A}$ & $\mathbf{C}$ & $\mathbf{A}$ & $\mathbf{B}$ & $\mathbf{C}$ \\
\hline Young & Total & $270.869 \pm 70.830$ & $264.823 \pm 50.235$ & $247.067 \pm 50.477$ & $86.504 \pm 3.965$ & $99.139 \pm 8.358$ & $88.268 \pm 7.415$ \\
& M1 & $258.458 \pm 83.422$ & $243.390 \pm 75.894$ & $235.239 \pm 59.953$ & $94.519 \pm 9.360$ & $101.522 \pm 19.698$ & $93.791 \pm 10.480$ \\
& M2 & $84.522 \pm 14.058$ & $87.059 \pm 19.501$ & $88.965 \pm 22.004$ & $46.799 \pm 7.466$ & $48.141 \pm 11.532$ & $47.585 \pm 8.937$ \\
& M3 & $184.082 \pm 25.588$ & $213.283 \pm 16.517$ & $190.809 \pm 25.685$ & $115.573 \pm 11.070$ & $130.126 \pm 11.114$ & $119.693 \pm 10.816$ \\
\hline Elderly & Total & $189.973 \pm 27.832$ & $213.509 \pm 21.026$ & $213.564 \pm 45.475$ & $76.358 \pm 3.203$ & $85.410 \pm 3.122$ & $77.770 \pm 7.078$ \\
& M1 & $188.168 \pm 27.811$ & $212.000 \pm 20.270$ & $213.564 \pm 45.475$ & $81.126 \pm 5.774$ & $87.280 \pm 3.075$ & $82.372 \pm 8.326$ \\
& M2 & $66.064 \pm 6.977$ & $67.977 \pm 18.067$ & $57.432 \pm 9.937$ & $39.860 \pm 6.977$ & $38.683 \pm 7.441$ & $36.246 \pm 6.136$ \\
& M3 & $134.086 \pm 33.163$ & $165.232 \pm 33.123$ & $140.901 \pm 30.023$ & $94.773 \pm 23.062$ & $110.738 \pm 22.902$ & $97.650 \pm 23.442$ \\
\hline
\end{tabular}

of foot (Total) by decreased $11.02 \%$, forefoot (M1) by decreased $11.24 \%$, midfoot (M2) by decreased $2.81 \%$ and rearfoot (M3) by decreased $18.85 \%$. d) The maximum mean pressure force of foot (Total) by decreased $10.60 \%$, forefoot (M1) by decreased $7.05 \%$ and rearfoot (M3) by decreased $14.42 \%$, while the maximum force of midfoot (M2) by increased 3.04\%.

2) Type $C$ (smart walking shoes)

a) The contact area of foot (Total) by increased $7.08 \%$, forefoot (M1) by increased 5.62\%, midfoot (M2) by increased $17.14 \%$ and rearfoot (M3) by increased 3.12\%. b) The maximum force of foot (Total) by decreased $1.47 \%$, rearfoot (M3) by decreased $7.37 \%$, while the maximum force of forefoot (M1) by increased $0.19 \%$ and midfoot (M2) by increased $24.15 \%$. c) The peak pressure of foot (Total) by increased $0.03 \%$, forefoot (M1) by increased $0.74 \%$, while the peak pressure of midfoot
(M2) by decreased $15.51 \%$ and rearfoot (M3) by decreased $14.73 \%$. d) The maximum mean pressure of foot (Total) by decreased $8.95 \%$, forefoot (M1) by decreased $5.62 \%$, midfoot (M2) by decreased $6.30 \%$ and rearfoot (M3) by decreased $11.82 \%$.

As a result of analysis, it has been found that Type A and Type $C$ have lower foot pressure (Total, M3) than Type B. Also, Type A and Type C show superior performance compared to Type B in all mask at contact area. Type A and Type $C$ shoes will be used to reduce foot pressure and increase comfort and fitting.

Published: 8 April 2014

\section{References}

1. Jason K Gurney, Uwe G Kersting, Dieter Rosenbaum: SBetween-day reliability of repeated plantar pressure distribution measurements in a normal population. Gait \& Posture 2007, 27:706-709. 
2. Cavanagh PR, Simoneau GG, Ulbrecht JS: Ulceration, unsteadiness, and uncertainty: the biomechanical consequences of diabetes mellitus. Journal of Biomechanics 1993, 26(11):23-40.

doi:10.1186/1757-1146-7-S1-A121

Cite this article as: Park et al:: Biomechanical analysis of smart walking shoe sending movement information to display device by radio communication. Journal of Foot and Ankle Research 2014 7(Suppl 1):A121.

Submit your next manuscript to BioMed Central and take full advantage of:

- Convenient online submission

- Thorough peer review

- No space constraints or color figure charges

- Immediate publication on acceptance

- Inclusion in PubMed, CAS, Scopus and Google Scholar

- Research which is freely available for redistribution

Submit your manuscript at www.biomedcentral.com/submit
Ciomed Central 